\title{
Renaissance des vignobles nivernais
}

Revival of vineyards in Nivernay

Wiedergeburt der Weinberge im Nivernais

\section{Marie-Pierre Cerveau}

\section{OpenEdition \\ Journals}

Édition électronique

URL : http://journals.openedition.org/rge/1328

DOI : $10.4000 /$ rge. 1328

ISSN : 2108-6478

\section{Éditeur}

Association des géographes de l'Est

\section{Édition imprimée}

Date de publication : 1 janvier 2006

ISSN : 0035-3213

\section{Référence électronique}

Marie-Pierre Cerveau, «Renaissance des vignobles nivernais », Revue Géographique de l'Est [En ligne], vol. 46 / 1-2 | 2006, mis en ligne le 18 décembre 2009, consulté le 08 septembre 2020. URL : http:// journals.openedition.org/rge/1328; DOI : https://doi.org/10.4000/rge.1328

Ce document a été généré automatiquement le 8 septembre 2020

Tous droits réservés 


\title{
Renaissance des vignobles nivernais
}

\author{
Revival of vineyards in Nivernay \\ Wiedergeburt der Weinberge im Nivernais
}

Marie-Pierre Cerveau

\section{Des vignobles anciens...}

1 Les vignobles nivernais ont acquis, pour certains d'entre eux, leurs lettres de noblesse au Moyen-Âge : les vins charitois rivalisent alors avec ceux de l'Auxerrois, de l'Orléanais ou d'Ile de France ; les vins de Tannay, acheminés par l'Yonne jusqu'à Paris, profitent d'une certaine notoriété. Le vignoble de " Ryosse ", inscrit sur la commune de Livry, au Sud du département actuellement (jusqu'en 1697, il appartenait à l'élection de Moulins et dépendait des Bourbons), né sous l'influence de Pierre II de Bourbon et d'Anne de Beaujeu, fille du roi Louis XI, bénéficie lui aussi d'un prestige certain. Tous les vignobles nivernais enregistrent une expansion et leur apogée au XIX ${ }^{e}$ siècle. Ainsi, les communes inscrites autour de La Charité-sur-Loire couvrent 693 ha en 1788, 1384 ha en 1874 et 1643 ha en 1886 ; cette extension est liée à la plantation massive de Gamay, qui favorise des rendements importants, mais peu qualitatifs.

2 En 1860, lors du recensement effectué par le docteur Guyot, chargé d'établir l'inventaire des vignobles de France, à la demande de Napoléon III, tous les villages du département, à l'exception des communes morvandelles et celles situées en fond de vallée, possèdent des vignes, d'étendue, certes, disparate. Livry compte alors 300 ha de vignes, exploitées par 300 vignerons.

3 Le sous-préfet Marlière recense les activités de l'arrondissement de Clamecy, où il est en poste en 1860. A cette époque, le vignoble s'étend sur 3157 ha, ventilés sur les six cantons de l'arrondissement. Les cantons de Tannay, Brinon-sur-Beuvron et Clamecy regroupent l'essentiel (près des $3 / 4$ de la surface viticole de l'arrondissement), avec respectivement 861,9, 731,4 et 715,7 ha de vignes, soit 2309 ha, comme le montre la carte. Certaines communes présentent de vastes superficies encépagées, ainsi Varzy (212 ha), Tannay (près de 124 ha), Metz-le-Comte (108,85 ha)... En 1860, les vignes 
couvrent 3 à $6 \%$ des superficies imposables dans de nombreuses communes, dont Clamecy (4,94\%), Dornecy (5,4 \%), Varzy (5,8 \%), Corbigny (5,99\%). Les cantons de Brinon et de Tannay enregistrent des pourcentages nettement supérieurs - 8 à $13 \%-$ dans plusieurs communes. Ainsi, à travers le canton de Brinon-sur-Beuvron, les vignes totalisent 10,22 \% de la superficie imposable à Brinon-sur-Beuvron, $13 \%$ à Asnan. Cinq communes du canton de Tannay dépassent $8 \%$ : Tannay $(8,41 \%)$, MoissyMoulinot (9,18 \%), Lys (9,42 \%), Asnois (11,67 \%) et Flez-Cuzy (11,77\%). Cette activité est pratiquée au sein d'une polyculture, où figurent élevage et petite céréaliculture. La viticulture apporte des revenus complémentaires aux familles paysannes ; elle est aussi destinée à l'autoconsommation.

Figure 1 : Répartition des superficies viticoles à travers l'arrondissement de Clamecy en 1860, d'après l'inventaire du sous-préfet Marlière.

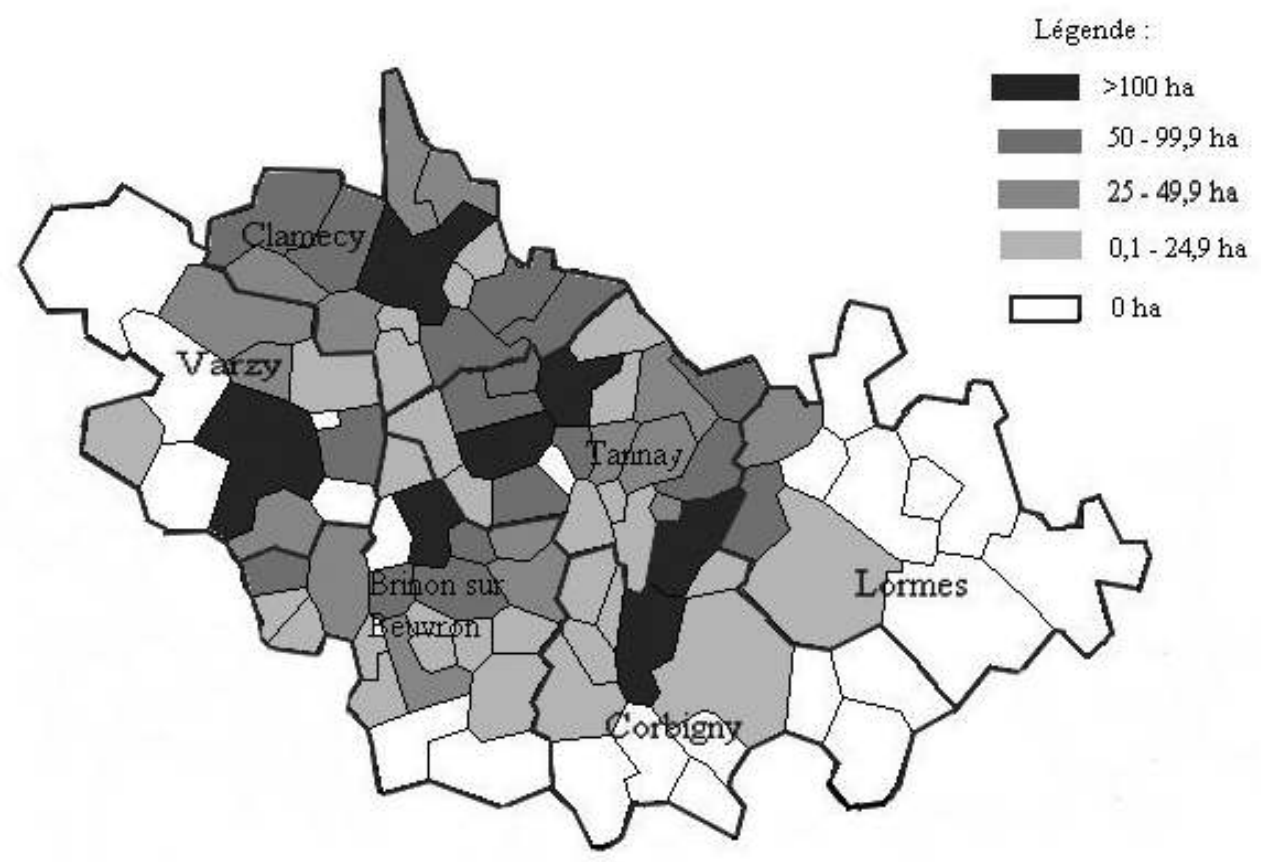

Les communes viticoles disposent de coteaux bien orientés au Sud-Est, au Sud ou au Sud-Sud-Ouest. Les sols reposent au Nord-Ouest et à l'Ouest de notre aire d'étude sur des strates argilo-calcaires supraliasiques ou marno-calcaires (Oxfordien, Kimméridjien $\mathrm{du}$ Jurassique supérieur), identiques à celles du Chablisien au Nord, de Pouilly et du Sancerrois à l'Ouest. Le vignoble de Livry s'étend sur des strates argilo-calcaires gréseuses du Rhétien (étage triasique), séparées des argilo-calcaires de l'Hettangien et du Sinémurien (étages liasiques) par une faille. Les cantons de Corbigny et Lormes, inscrits en partie sur le massif granitique du Morvan, le canton de Varzy, boisé et traversé par des vallées où les risques de gelée sont tardifs, présentent ponctuellement des aires viticoles.

5 À la fin du XIX siècle, les principaux cépages utilisés sont, pour les vins rouges, les Gamay, Pinot, Teinturier, et pour les blancs, les Chardonnay, Sauvignon et Melon dans la sphère de Tannay. Une partie de ces vins était destinée aux cafetiers des villes voisines, des villages et à la consommation familiale. Les fermentations souvent mal maîtrisées, la conservation dans des futailles de qualité médiocre, les caves à l'hygiène réduite, donnaient des vins peu aptes à la conservation. La froidure hivernale 
conservait les vins, mais dès le début du printemps, la fermentation reprenait, d'où la sensation de pétillement du vin. Au-delà de cette période, le vin ressemblait à une piquette.

\section{... Mais en déclin de la fin du XIXe aux années 1980}

6 L'extension maximale des surfaces encépagées est atteinte en 1885-1890 : à Tannay, la vigne couvre alors 184 ha. Le phylloxéra touche distinctement les vignobles nivernais : si Riousse est peu affecté, les vignobles des arrondissements de Clamecy, Cosne, Pouilly et La Charité sont décimés. Dans le Tannaysien, les premiers symptômes du phylloxéra apparaissent en 1885 ; entre 1890 et 1905, la propagation est foudroyante. En 1905, les vignes couvrent 25 ha à Tannay, soit une chute de $85 \%$ en 20 ans. Dans le Charitois, le vignoble, d'abord confronté au mildiou entre 1886 et 1888, est détruit par le phylloxéra entre 1890 et 1894. En 1899, la vigne couvre 64 ha ; 1579 ha, soit 96 \% du vignoble, ont disparu.

7 Une fois le diagnostic du phylloxéra établi, les viticulteurs arrachent les ceps contaminés, la mort dans l'âme. Certains ne pourront survivre à cette grave crise qui ruine de nombreuses familles, propriétaires ou employées, vivant de la viticulture et accélère l'exode rural amorcé dès la seconde moitié du XIX ${ }^{e}$ siècle vers Paris. Quand ils restent au pays, les hommes se louent dans les fermes environnantes pour effectuer les travaux saisonniers (fenaison, moisson), travaillent dans les forêts (le bois de chauffage est toujours acheminé vers Paris par l'Yonne) ; les femmes travaillent à façon pour l'industrie textile ou font des ménages dans les environs. Dans tous les cas de figure, la survie est difficile. La misère touche de nombreuses familles.

Néanmoins, une poignée d'hommes acharnés reconstitue les vignobles. Fin XIX ${ }^{e}$, un syndicat de défense des vignerons est créé à La Charité, où des ceps sont replantés. En 1912, 892 ha couvrent de nouveau les coteaux charitois. La Première Guerre Mondiale stoppe cet élan, retarde d'autant la replantation et prolonge le déclin des vignes nivernaises. Le vignoble de Pouilly-sur-Loire, dont nous n'analyserons pas les destinées ici, constitue une exception. Reconstitué dans des conditions socio-économiques difficiles, il bénéficie, comme le vignoble sancerrois, d'un réencépagement en Sauvignon et en Chasselas. Ce choix sauve la vie des familles vigneronnes. Les vins issus de ces cépages sont de bonne qualité et l'INAO lui attribue l'AOC en 1937.

9 Dans les autres vignobles, durant l'Entre-deux-guerres, les hybrides remplacent les cépages greffés et accélèrent le déclin de l'activité viticole. Ainsi, le vignoble charitois enregistre une baisse constante entre 1918 et $1939: 445$ ha en 1929, 384 ha en 1939. Le choix des hybrides ne pardonne pas lors des difficultés économiques enregistrées au cours des années 1950 dans le monde viticole. En effet, la forte concurrence émanant des vins du Midi sur le marché des vins de consommation courante, adjointe à l'amélioration qualitative des vins commercialisés, provoque la dépréciation gustative des vins issus des hybrides et la baisse inéluctable de leur consommation. La prime à l'arrachage précipite la disparition d'une partie des vignes. Dans le contexte de l'agriculture productiviste, les vignerons se convertissent à l'élevage ou à la céréaliculture.

10 Demeurent quelques parcelles viticoles cultivées pour l'autoconsommation et, plus rarement, à des fins commerciales. Entretenus avec soin, souvent complantés de pêchers, ces arpents de vignes sont la fierté de leur propriétaire. Les Anciens s'en 
occupaient, mais leurs enfants, partis travailler en ville, ne désirent pas entretenir ce patrimoine familial emprunt de désuétude, nécessitant du travail et donnant des vins éloignés des standards à la mode. Ce vignoble, émietté et disséminé à travers l'espace nivernais, s'est maintenu jusque dans les années 1960. En 1960, seuls persistent 30 ha de vignes sur la commune de Livry, auxquels le remembrement donnera le coup de grâce. La situation est identique dans les autres aires viticoles ; en 1964, le vignoble charitois couvre 120 ha.

11 Pour autant, durant les années 1960, le temps d'une journée, les vendanges rassemblent encore ; c'est l'occasion de se retrouver autour d'une table bien garnie. En fait, les vendanges servent de prétexte pour réunir famille et amis, associer travail et convivialité. Gibier fraîchement tué lors de l'ouverture de la chasse, coq au vin, œufs en meurette ${ }^{1}$, fromages maison exhalant des odeurs décriées désormais par les partisans d'une hygiène excessive, îles flottantes et croquets (spécialités de La Charité, Donzy et Varzy) font la joie des convives et sont la fierté de la maîtresse de maison, trop heureuse de montrer ses compétences culinaires. C'est aussi pour le maître de maison le jour de sortir de sa cave un Sauvignon de Pouilly, plus rarement un Bourgogne, trop cher pour des familles aux revenus encore modestes.

Les vignes ont été arrachées durant les années 1960-1970, quand les communes ont été remembrées. À la même époque, nos aïeux, trop âgés, ne pouvant plus subvenir à leur entretien, ont laissé faire la jeune génération, poussée par la recherche de la productivité, pour étendre prairies ou terres labourables. La vigne, grande mangeuse de temps et non rentable, ne peut entrer dans le schéma de l'agriculture moderne. Les vignobles nivernais poursuivent leur inexorable déclin. Ainsi, en 1980, le vignoble charitois, quasiment moribond, couvre 20 ha.

Les épisodes climatiques froids des hivers 1984-85, 1985-86 (- 25 à $\left.-35{ }^{\circ} \mathrm{C}\right)$ marquent l'arrêt de mort de ces vignes. À Livry, en 1990, restent 3 ha de vignes. Un pan de l'histoire des campagnes nivernaises disparaît durant cette période. Néanmoins, le vignoble demeure vivace dans la mémoire collective et des hommes sont prêts à le faire revivre.

\section{La renaissance : une dynamique insufflée de l'intérieur ou/et de l'extérieur}

14 Il faut attendre les années 1980-90 pour voir des vignes remplacer les friches. Quatre aires viticoles renaissent : au centre-Ouest dans les environs de La Charité-sur-Loire, au Nord-Ouest, près de Cosne-sur-Loire, au centre-Nord, autour de Tannay et au Sud, à Livry. Ces vignobles n'ont jamais totalement disparu, il suffisait de raviver des pratiques ancestrales. Ils bénéficient de savoir-faire, certes empiriques, mais l'acharnement de quelques hommes permet d'envisager un nouveau départ.

\section{A. Une renaissance initiée in situ pour les vignes ligériennes (centre- Ouest et Nord-Ouest)}

\section{La fougue des Charitois, relayée par des vignerons exogènes}

15 La renaissance viticole est amorcée dans la région de La Charité. En janvier 1980, l' Union viticole de La Charité est créée à l'initiative de quelques vignerons bien 
déterminés dans leur démarche. Ces hommes refusent de voir un patrimoine disparaître dans la tourmente socio-économique. Ils ont soigneusement conservé un encépagement de qualité sur les coteaux bien exposés et préparé un dossier afin d'accéder à la dénomination en Vin de Pays de zone.

La reconnaissance parvient par le décret du 22 janvier 1986. Celui-ci délimite une aire de dénomination, circonscrite à La Charité et à cinq communes limitrophes - Chasnay, Nannay, La Celle-sur-Nièvre, Raveau et Parigny-lesVaux - et donne vie aux Vins de Pays des Coteaux Charitois. Une nouvelle aventure commence. Chardonnay et Pinot noir remplacent le Sauvignon et le Gamay trop productif. Les vignes, plantées sur les strates du Jurassique moyen aux sols argilo-calcaires, sont bien exposées (SudOuest, Sud-Sud-Ouest).

Le succès, enregistré par les vignerons, attire les investisseurs. Les vignerons de Pouilly trouvent dans le vignoble de La Charité des terres en friches, certes, mais à des tarifs défiant toute concurrence. De surcroit, le terroir présente de belles qualités. Serge Dagueneau de Saint-Andelain fait arracher des bois et planter des vignes sur les hauteurs de Chasnay. La Cave des Hauts de Seyr est créée à son initiative, sous forme d'un GFA, Groupement Foncier Agricole et capte une centaine de personnes. Les vignerons adhérents vendent directement leur récolte à la Cave sans avoir à se préoccuper de la vinification, de l'élevage, de la comptabilité. La maitrise technique de la vinification, d'abord confiée à Dagueneau, permet à la Cave des Hauts de Seyr de commercialiser dès 1995 des vins blancs de bonne qualité et des rouges honorables. Ensuite, la Cave engage un œnologue.

Actuellement, les domaines présentent des tailles diverses, de quelques ares à plus de 10 ha. Les vins produits sont eux aussi fort disparates en terme de qualité. Parmi les exploitations, le Domaine du Puits de Compostelle ${ }^{2}$ se détache nettement du lot. Imaginée en 1998 par un œnologue et deux de ses amis, cette exploitation prend vie en 1999. Emmanuel Rouquette achète une parcelle dénommée La Belle Catherine, sise sur la commune de Nannay. Les premières vendanges ont lieu dès 1999. Le vigneron s'installe à Mauvrain sur la commune de La Celle-sur-Loire. En mars 2000, il crée une SCEA (Société Civile d'Exploitation Agricole) au sein de laquelle adhère une dizaine de personnes, amis et membres de la famille du vigneron. L'exploitation continue de se développer au grand bonheur de ses clients. L'aire des Coteaux Charitois couvre désormais plus de 40 ha et compte 16 producteurs en plus de la Cave des Hauts de Seyr. Parmi ces exploitations, nous dénombrons 3 GAEC, 2 SCEA et 11 vignerons indépendants. En 2004, les communes de La Charité, Chasnay, Nannay et La Celle-surNièvre fournissent $90 \%$ de la production. Cette dernière est ventilée entre les Chardonnay (48\%), les Pinot noir (25\%), le Sauvignon (15\%) et le Gamay (12\%).

La proximité de Pouilly et de Sancerre, l'inscription de l'aire de dénomination en Vins de Pays des Coteaux Charitois dans une région très touristique (La Charité devient Patrimoine mondial de l'UNESCO en décembre 1999) dynamisent le vignoble des Coteaux Charitois. Les caves ouvertes par les vignerons à La Charité et dans les communes environnantes, le Syndicat d'initiative, les restaurateurs et les festivals (musique à La Charité, cinéma à Nannay) donnent à connaître cette aire viticole. 


\section{Une nouvelle vie instillée par des acteurs multiples, le cas des Coteaux du Giennois}

Parallèlement, durant la décennie 1980, une nouvelle vie viticole naît au Nord-Ouest du département. Le vignoble, inventorié en VDQS ${ }^{3}$ depuis 1954, s'étend sur les communes d'Alligny-Cosne, La Celle-sur-Loire, Myennes, Neuvy, Cosne-sur-Loire, Pougny, SaintLoup et Saint-Père. La viticulture, relictuelle, est, dans cet espace, une activité complémentaire à la céréaliculture.

L'extension du vignoble des Coteaux du Giennois est liée à plusieurs paramètres : la Cave de Pouilly-sur-Loire produit des Coteaux du Giennois depuis 1970 et obtient des résultats satisfaisants ; le lycée agricole de Cosne crée une section viticole pour répondre à la forte demande émanant des vignobles de Pouilly et Sancerre et à une demande croissante provenant des Coteaux du Giennois ; l'Aoc Coteaux du Giennois est attribuée par l'INAO, par décret du 15 mai 1998, sur un espace étendu de Gien à Saint-Père et Pougny dans la Nièvre.

Figure 2 : Aire de dénomination (Vins de Pays) et d'appellation (AOC) des vignobles nivernais

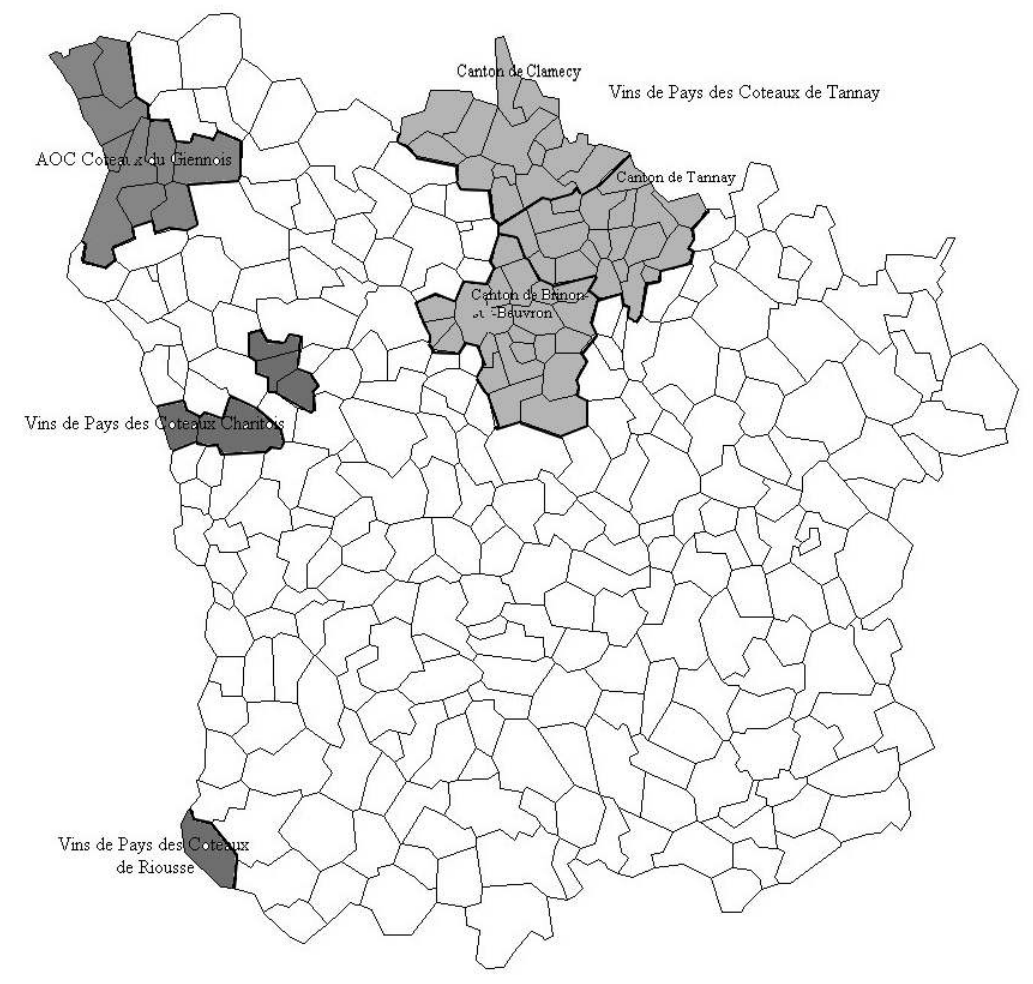

Grâce à l'appui de la Cave de Pouilly-sur-Loire, les vignerons du Giennois peuvent espérer une meilleure connaissance de leurs vins : par sa structure, la Cave dispose d'une large clientèle, qui plus est, cosmopolite. Grâce à l'outil pédagogique fourni par la section viticole du lycée agricole, les jeunes bénéficient de bonnes bases de travail. L'AOC allouée à cet espace est perçue comme un coup de pouce, moins généreux qu'il n'y paraît, au vu du contexte concurrentiel. Il existe donc une triple dynamique dans l'extension des Coteaux du Giennois, associée à la proximité des vignobles moteurs de Sancerre et Pouilly, où la jeune génération effectue des stages et prend modèle sur les vignerons dont la réussite socio-économique est patente depuis 30 ans. 
23 À Cosne, Saint-Père et Pougny, des jeunes reprennent les vignes de leurs pères et désirent travailler autrement. Certains choisissent d'étendre la superficie viticole familiale, mécanisent les travaux, investissent dans des pressoirs modernes. D'autres élisent la voie de la viticulture biologique, à une époque (années 1980) où cette activité, très marginale dans la sphère agricole, l'est encore plus dans le monde viticole. Dans tous les cas, la viticulture est pratiquée en tant que monoactivité et à des fins commerciales.

24 Aucune cave coopérative n'est créée dans cet ensemble viticole. Les initiatives, privées, émanent de quelques familles vigneronnes qui n'ont jamais cessé leur activité. Elles travaillent à Cosne, Saint-Père et Pougny. Dans chacune de ces deux dernières communes, un producteur a choisi de pratiquer la viticulture biologique. À Villemoison, sur la commune de Saint-Père, Alain Paulat a repris le domaine de son père. Les vignes sont enherbées, labourées (buttées et débuttées), sarclées et vendangées à la main. Il cultive des Gamay et des Pinot noir. Les deux cépages sont assemblés pour donner deux cuvées : Les Belles Fornasses, à majorité Gamay, Les Têtes de Chat à majorité Pinot noir. Il pratique des cuvaisons de 15 jours, la vendange est foulée, peu ou pas égrappée. Les levures sont indigènes. L'élevage est pratiqué dans des cuves inox, puis, durant 6 à 8 mois, en fûts de chêne anciens. Les vins, non collés, sont légèrement filtrés (sur des filtres en silice) avant la mise en bouteille. La production est étonnante au sens qualitatif du terme. Les vins, mis à vieillir pour une durée minimale de 5 ans, prennent des teintes tuilées aux reflets mordorés et ont des accents bourguignons marqués. Leur mode d'expression très original les fait se démarquer nettement des autres vins issus des Coteaux du Giennois.

Au début des années 2000, un nouveau type de producteurs s'adjoint aux vignerons de cette aire. Voyant le succès des AOC Coteaux du Giennois, les frères Thibaut, céréaliers intervenant sur des exploitations de plusieurs centaines d'ha, créent une société viticole, le Domaine de Villargeau,planté en Pinot noir, Gamay et Chardonnay. L'approche du travail dans les vignes et en cave, les objectifs économiques diffèrent de ceux des vignerons voisins : les Thibaut se positionnent dans le schéma d'une exploitation rentable et scientifique. En trois ans, le domaine est passé de 10 à 17 ha. Le travail y est largement mécanisé, les vignes sont désherbées et traitées chimiquement. Afin d'optimiser les vendanges, l'élevage et la commercialisation de leurs vins, les frères Thibaut ont construit une cave adaptée à leur production, très bien implantée et signalée sur la route reliant Clamecy à Cosne, à moins de 10 minutes de l'A77. Un des fils s'occupe désormais exclusivement de cette exploitation viticole.

\section{B. La renaissance du vignoble tannaysien ou l'intervention d'acteurs exogènes}

Au milieu des années 1980, l'arrondissement de Clamecy, a, quant à lui, perdu la quasi totalité de son vignoble. Le canton de Clamecy présente des vignes éparpillées, entretenues par des vignerons amateurs, à des seuls fins d'autoconsommation. Quelques arpents subsistent à Oudan, Courcelles... Villiers-sur-Yonne préserve son patrimoine viticole, inscrit au lieu-dit Vautoins, et ses traditions vini-viticoles (SaintVincent), tout comme le village voisin d'Asnois. Dans ces deux villages, les plants hybrides constituent la totalité de l'encépagement. Tannay, village réputé pour ses vins blancs issus du cépage Melon, est la seule commune à avoir conservé une partie de ses 
vignes. Néanmoins, le temps des 184 ha est loin. Seuls quelques vignerons défendent un héritage en perdition : les jeunes sont davantage attirés par la ville, un seul négociant achète la production viticole de médiocre qualité du village et des communes alentours. La venue d'un homme extérieur à la région tannaysienne, Pierre Hervé (il est d'origine parisienne) et au monde viticole (il est instituteur) modifie la perception des terres à vignes repassées à l'état de friches dans les années 1960-1980. Cet homme a entrepris des études viticoles (BEPA, BTSA) à Mâcon-Davayé. Parallèlement, il tente d'acheter des droits de plantation. Son but est de produire des vins issus de vignes biologiques. Il visite les anciens propriétaires, méfiants, pour certains, de voir un étranger s'intéresser à des bouts de friches, trop heureux, pour d'autres, de penser à la renaissance du vignoble local. Progressivement, après de longues démarches, laborieux travaux d'arrachage de friches, 6 ha de vignes sont plantés en Chardonnay et Melon pour les cépages blancs et en Pinot noir pour les rouges sur trois communes - Villiers-surYonne, Brèves et Asnois - de la fin des années 1980 au milieu de la décennie suivante. Le Domaine de Bel Air est constitué en SCEA, au sein de laquelle participent famille et amis du vigneron.

Les vignes sont plantées en lyre (vignes hautes, soutenues par des piquets plantés en V), comme celles des Hauts de Côte de Nuits et de Beaune. Elles sont enherbées, buttées au printemps, puis débuttées (décavaillonées), plus tard. Du guano est apporté tous les 3 ou 4 ans, en fonction des besoins. La vendange est faite manuellement par des bénévoles réunis le temps de deux week-ends pour effectuer ce travail dans la bonne humeur. C'est aussi l'occasion de faire la fête (le vigneron est musicien à ses heures), comme le faisaient les Anciens. Les rouges sont égrappés. Deux pressoirs horizontaux effectuent une pressée douce, afin d'éviter la production de vins astringents. Certains vins sont élaborés comme les Beaujolais : les raisins entiers sont mis en cuve de résine durant une dizaine de jours, en fonction de la température et de la transformation du sucre en alcool. À l'issue de la cuvaison, les vins rejoignent la cave, où ils vieillissent en fûts de chêne anciens durant 12 à 18 mois. Cet élevage long permet aux vins de prendre des saveurs délicates. Les blancs sont soit élevés en fûts de chêne anciens, soit en cuve de résine, c'est le cas des Melon (appréciés dans la région), de certains Chardonnay et Pinot noir. Les vins obtenus ont des ressemblances avec les vins de Côte d'Or, où le vigneron a effectué ses stages. Les Pinot, plus ou moins tanniques, présentent des arômes de fruits rouges (feuilles de cassis, framboise), parfois doublés d'effluves de vieux cuir ou de sous-bois. Les blancs issus du Chardonnay s'affichent dans le registre des fleurs blanches (acacia), des fruits secs et du beurre frais.

Les vignerons amateurs viennent voir les vignes du néo-vigneron, se renseignent et essaient de copier. Ils arrachent progressivement les hybrides et les remplacent par du Melon, des Chardonnay et des Pinot noir (Vézelay n'est pas loin et la vigne lui réussit). Néanmoins, quelques vignerons amateurs entretiennent toujours des hybrides donnant des vins à la mâche rugueuse.

À son arrivée dans la région, Pierre Hervé constitue un dossier afin d'obtenir la dénomination en Vins de Pays de zone pour l'aire du vignoble de Tannay ; cela a pour effet de dynamiser et de régénérer ce vignoble endormi. Il prolonge sa démarche avec la création des Caves Tannaysiennes, constituées en SARL, en compagnie de Cyrille Raty en 1995. Cette cave, installée dans d'anciens locaux ecclésiastiques, le Cellier des Moines, est restaurée avec l'aide du Conseil général et dotée d'infrastructures modernes : cuveries, pressoirs, futaille. 
31 Le vigneron-instituteur, qui a vite cessé son partenariat avec la Cave de Tannay, se fait pédagogue auprès de ses collègues pour les inciter à améliorer la conduite viticole dans les vignes et en cave. Il s'agit avant tout d'éviter la reproduction de connaissances empiriques et de favoriser des méthodes plus scientifiques. Le travail dans les caves s'affine : les analyses se systématisent afin de doser au mieux les apports en sucre (chaptalisation), en soufre, afin d'intervenir à temps sur les vins en fermentation. L'amélioration qualitative généralisée des vins conduit l'ONIVINS à répondre à la demande de Tannay et à lui attribuer la dénomination Vins de Pays des Coteaux de Tannay par le décret du 21 janvier 2001. Les communes inscrites dans les cantons de Clamecy, Tannay et Brinon-sur-Beuvron peuvent alors bénéficier de cette dénomination.

P. Hervé, devenu viticulteur à plein temps, ne peut cependant infléchir la propension qu'ont les vignerons de la contrée à avoir la main lourde sur les traitements chimiques. En 2000, printemps et été très humides et chauds génèrent le développement de maladies cryptogamiques et amènent les vignerons à traiter pour éviter la prolifération entre autres du mildiou. Quand les feuilles des vignes traitées chimiquement tombent bien avant les vendanges, la bonne santé des vignes conduites en viticulture biologique, passées à la bouillie bordelaise, amène les vignerons à s'interroger sur l'arsenal chimique utilisé dans leurs vignes. Mais sans que cela ne serve de leçon...

Des vocations se sont découvertes dans cette région depuis ces dix dernières années. Ainsi, à Asnois, le menuisier Jacky Gobillot a développé une petite exploitation viticole de 0,5 ha. Il envisage d'y consacrer tout son temps à la retraite. Il a acheté un pressoir, utilisé par tous les producteurs du village, le temps des vendanges. Cette mutualisation du matériel s'accompagne d'une entraide en terme de connaissances vini-viticoles. Ici, pas de compétition entre les producteurs, chacun apporte son savoir et cherche à en faire bénéficier le voisin. Cette politique porte ses fruits et l'ambiance villageoise s'en ressent. Cette dernière est perceptible en terme de chaleur humaine lorsqu'il s'agit de visiter les caves des uns et des autres.

De leur côté, les Caves Tannaysiennes attirent quelques investisseurs : 5 personnes physiques et 5 vignerons locaux. Les producteurs s'engagent à livrer leur récolte, la cave à élaborer le vin. Un œnologue, Cyril Ponnelle, est engagé pour veiller à la production de vins de qualité. Le challenge est difficile car les agriculteurs-vignerons recherchent les rendements élevés. L'œnologue désire améliorer la qualité des vins pour que la production, devenue importante, puisse être aisément commercialisée. En 2000 , le cahier des charges instaure une grille tarifaire récompensant la qualité des raisins sains parvenus aux Caves, mais son application s'avère difficile...

En 2004, la superficie en production couvre 17,2 ha, ventilée entre les Chardonnay $(8,66$ ha 66), Melon (5,24 ha), Pinot noir ( $2,7 \mathrm{ha}$ ) et Gamay ( $0,79 \mathrm{ha})$. La production totale s'élève entre $352 \mathrm{hl}$, soit 47000 bouteilles (en 2003, année de fortes chaleurs) et 1125 hl, soit 105000 bouteilles en 20045. La politique des Caves vise à régénérer l'exploitation viticole : les vieux ceps, plantés dans les années 1970, sont arrachés et remplacés par des cépages nobles, il en est de même pour les parcelles encépagées en hybrides. À terme, il est prévu d'étendre le vignoble des Caves à 22-25 ha.

La culture des vignes est conduite en parcelles désherbées et traitées chimiquement. Les vendanges, manuelles, emploient 22 à 25 personnes durant 8 à 14 jours, selon la récolte. Les Caves Tannaysiennes utilisent des pressoirs horizontaux. L'embouteillage est effectué par un prestataire de services. L'appartenance à l'aire de 
dénomination en Vins de Pays de zone annonce l'étape suivante, celle de l'appellation d'origine en VDQS.

\section{Le vignoble de Riousse dynamisé par des intervenants institutionnels, épaulés par des professionnels de la vigne}

Parallèlement, d'autres intervenants mettent un point d'honneur à valoriser leur patrimoine viticole. Ainsi, en 1992, la municipalité de Livry, sous la houlette du maire, conseiller général, Christian Barle, crée une association pour réhabiliter le vignoble de Riousse. Elle lance un appel à souscription auprès de la population afin de trouver des bases financières pour fonder la SCEA du Clos de Riousse et dynamiser l'activité sans craindre un trop lourd investissement. 600 actionnaires répondent à cette initiative $^{6}$. Les parts sont vendues à l'époque $1000 \mathrm{~F}^{7}$. En 1995, la commune obtient la dénomination en Vins de Pays de Riousse.

Depuis 2000, la SCEA est propriétaire d'un domaine de 16 ha, dont 15 ha encépagés en Gamay (30\%), Pinot noir (37 \%) et Chardonnay (33\%). Des bâtiments anciens sont réhabilités dans le hameau de Riousse pour accueillir une cuverie moderne et un musée du Vin, ouvert durant la période estivale. Une cave est construite afin d'élever les vins rouges en fûts de chêne.

Les travaux dans les vignes sont effectués par un œnologue, un vigneron formé sur le tas, auxquels s'adjoint un prestataire de services pour la taille. Des sociétaires bénévoles viennent tirer les bois, accoler et vendanger manuellement. La vinification est confiée à l'œnologue. Les rendements sont compris entre 50 et $60 \mathrm{hl} / \mathrm{ha}$. La production s'élève à environ $1000 \mathrm{hl}$, soit 60000 bouteilles. L'embouteillage, longtemps confié à un embouteilleur sancerrois, est effectué depuis 2004 par La Chablisienne ${ }^{8}$, dont le sérieux est apprécié par les adhérents.

Les vins proposés sont des Chardonnay, des Pinot noir élevés en cuves ou en fûts anciens, des Gamay, des Rosés, des Gris (issus des bourbes de Chardonnay) à la robe saumonée pâle et, depuis 2000, du Pétillant de Riousse. Ce dernier, fabriqué selon la méthode champenoise, associe Pinot noir et Chardonnay, à proportion identique. En raison du manque d'infrastructures spécifiques, ce vin n'est pas vinifié sur place ; il est confié à Jean-Baptiste Clair, vigneron établi en Côtes Roannaises. Il plaît à la clientèle et chaque année 4000 bouteilles sont produites.

41 À la cave de Riousse, s'est adjoint un viticulteur indépendant, Fabrice Barle, qui, depuis 2000-2001, cultive 3 ha de vignes. Il travaille seul ; son père à la retraite, vient de temps à autre, l'aider. Ses vignes sont encépagées en Chardonnay et en Pinot noir.

\section{Marchés et rôle des médias}

\section{A. Commercialisation}

L'augmentation de la production viticole nivernaise nécessite un élargissement du marché et une attention toute particulière au maintien ou à l'amélioration qualitatif des vins commercialisés. Or, le moindre faux pas peut être fatal à toute l'aire de dénomination, d'où la nécessaire entente entre vignerons. Pour le moment, seuls les viticulteurs de la région de Tannay ont instauré des dégustations de cave en cave, 
comme cela se pratique ailleurs. Pour autant, les secrets sont jalousement gardés par les vignerons, qui craignent la concurrence à l'intérieur de leur aire viticole.

Les vignerons et les caves inscrites en SARL ou en GFA commercialisent une partie de leurs vins in situ. Ainsi, la Cave des Hauts de Seyr, bien située le long de la D151, passage obligé des touristes en direction de l'Atlantique, reçoit une partie de sa clientèle dans ses locaux. La commercialisation au domaine est très prisée des clients à la recherche du contact et du dialogue avec le vigneron. Cependant, les travaux viticoles obligent les viticulteurs à prendre rendez-vous avec leur clientèle particulière. Celle-ci est reçue à la cave afin de déguster les vins de l'année en élevage sur fût et les vins des années antérieures embouteillés. À Saint-Père, A. Paulat vend au domaine ; s'il est absent, ses parents, anciens vignerons, le remplacent. Il se rend aussi sur des foires. Il a choisi de faire des vins de qualité et il lui faut être patient : il commercialise des vins ayant effectué un vieillissement d'au moins cinq ans. Ses difficultés financières l'ont conduit à revoir sa stratégie et à commercialiser aussi des vins jeunes, vendus en fontaine à vin. La clientèle apprécie l'accueil à la cave, les produits proposés, les tarifs sages $^{9}$ et le rapport qualité/prix.

Dans le contexte d'un marché concurrentiel, les producteurs veillent à être présents dans un large rayon autour de leur domaine. Ils vont à la rencontre de la clientèle, fréquentent les marchés, foires et d'autres manifestations locales pour capter une clientèle qui ne viendrait pas acheter à la cave.

La Saint-Vincent permet également de faire connaitre les vins à des convives venus parfois de très loin. La région de Tannay, où la convivialité, fortement ancrée dans les traditions, honore Saint-Vincent dans différentes communes : messe, tournée apéritive dans les caves des vignerons, déjeuner haut en couleurs où les convives peuvent apporter leurs flacons, intronisation dans les confréries vineuses, concours des meilleurs vins, dégustations dans les caves des lauréats... Chaque vigneron veille à participer aux manifestations organisées par son village et à celles des communes voisines. Les festivités estivales (Fête de l'Andouillette et du Vin blanc à Clamecy,...) sont aussi l'occasion de proposer les vins de la région à la dégustation et à la vente. Les comices agricoles récompensent les meilleurs vignerons.

46 Toutes ces démarches nécessitent beaucoup de temps et posent problème en période végétative, quand la vigne requiert des interventions multiples : taille, accolage (attachage) des pampres... Le vigneron emploie alors un ouvrier pour l'aider à mener à bien ses travaux dans les vignes, sous peine de perdre une partie de sa récolte et d'entraver ainsi l'essor de son entreprise.

Les vignerons, adhérents ou actionnaires des Caves participent aux foires et salons pour faire connaitre les vins de leur domaine respectif, mais dans une conjoncture morose et une concurrence aiguisée, la tâche est ardue. Cette dernière s'avère délicate, quand les actionnaires ont des connaissances limitées sur les vins produits et ne peuvent répondre aux questions posées par les visiteurs (cas de la Cave de Riousse). Les Caves Tannaysiennes ont, quant à elles, revu leur stratégie commerciale : les vignerons adhérents présentent désormais les vins dont ils connaissent l'histoire, du cep à la vinification. Les non-producteurs participent aux manifestations commerciales à condition d'être accompagnés par un professionnel. Les Caves Tannaysiennes étaient présentes au Salon de l'Agriculture à Paris en 2005 pour nouer de nouveaux contacts et vendre leurs vins à d'autres clients. La communication est prise au sérieux par le vignoble de Tannay, comme le prouve le DVD réalisé sous la houlette de Cyrille 
Raty. Ce support vise à mieux faire connaître cette aire viticole, auprès des clients potentiels. Il fait intervenir Francis Salamolard, cuisinier à Quarré-les-Tombes (Yonne), qui valorise le Melon et le Ratafia à travers deux recettes appétissantes.

Outre la clientèle particulière, certains vignerons démarchent aussi les cavistes de proximité (Clamecy, Auxerre, Avallon, pour les vins des Coteaux de Tannay) ou plus lointains (région parisienne), les supermarchés, les restaurateurs. Cela demande de la disponibilité et requiert une grande finesse dans le choix d'une clientèle volatile. Parfois les cavistes initient la demande, quand ils ont lu des articles dans des revues spécialisées. Le recours à un commercial multicartes est nécessaire dès que la production s'avère trop importante à commercialiser par le domaine. Certains domaines ont confié la commercialisation à un professionnel, ainsi le Domaine de Villargeau (frères Thibaut) ${ }^{10}$, les Caves Tannaysiennes et des Hauts de Seyr. Cette démarche nécessite de bien cibler la clientèle. Les Caves Tannay ${ }^{11}$. En effet, elles ont démarché les restaurants régionaux et ignoré leur clientèle traditionnelle. Cette politique a montré ses limites. Les ventes ont stagné à 8-9 000 bouteilles, alors que les prévisions devaient atteindre 30000 cols en 2005.

Les restaurants locaux, les manifestations officielles font également la promotion des vins de proximité. Cela ne suffit pas toujours pour écouler l'ensemble de la production annuelle. Par ailleurs, certains restaurateurs se plaignent de la faiblesse de la production viticole émise par les domaines particuliers, car ils ne peuvent proposer leurs vins toute l'année à leur clientèle. Dans un autre registre, ils trouvent parfois les tarifs trop élevés et quand il faut appliquer un coefficient multiplicateur de 2,5-3, les vins arrivent sur la table à des prix inaccessibles pour une partie de la clientèle. Ainsi, il est difficile de proposer à la clientèle un Coteaux de Tannay à un tarif supérieur à certaines AOC bourguignonnes, fut-il justifié par des qualités gustatives.

Les vins nivernais sont essentiellement vendus en France. Nonobstant, quelques vignerons et les Caves commercialisent aussi leur production à l'étranger, mais de manière marginale et souvent par l'intermédiaire de connaissances vivant dans la région. Les Caves Tannaysiennes commercialisent $58 \%$ de leurs vins auprès des particuliers, dont 2 à $3 \%$ d'étrangers. La vente aux professionnels s'élève à $42 \%$ dont $2 \%$ en direction de l'Union européenne. Le Royaume-Uni, l'Allemagne et les Pays-Bas sont les principaux clients. Des visiteurs étrangers, surtout Anglais et Hollandais, demeurant dans la région, fréquentent aussi les caves. À Riousse, les Anglais achètent surtout des Blancs, les Hollandais ont un faible pour les Pinot, les Chardonnay et les Gris.

51 Outre l'édition de dépliants, les domaines s'inscrivent désormais sur la toile. Cette démarche, cependant marginale, est en fait l'apanage soit de passionnés du vin (Rouquette), soit d'hommes pressés de commercialiser leurs vins (Thibaut). Il s'agit soit de sites particuliers, soit de sites hébergés par des enseignes commercialisant du vin. Le Domaine du Puits de Compostelle (Rouquette) propose une promenade historique, des renseignements ${ }^{12}$. L'esthétisme est soigné et l'internaute peut passer un bon moment dans les vignes. Ce domaine est aussi hébergé par un site internet commercialisant des vins issus d'autres régions viticoles. Le Domaine de Villargeau, présent sur la toile, est hébergé par le site intitulé 1855 (date de classement des vins de Bordeaux). La visite est rapide : pas de présentation du domaine, fiche technique des deux vins mis en vente (blanc et rouge) réduite, seul est indiqué le tarif, 6,3 euros. Contrairement au Domaine du Puits de Compostelle, 
où la passion transparaît au fil des pages web, le Domaine de Villargeau n'invite guère à l'achat. Outre ces exploitations, Guy Saget, viticulteur à Pouilly propose également un site où figurent des vins du Giennois. Les Caves présentent aussi des pages Internet de qualité disparate. Si les Caves de Pouilly jouent le grand jeu médiatique, celles de Tannay n'ont pas encore plongé dans la sphère Internet.

Pourtant, cette dernière est un moyen très efficace pour faire connaître ces vins et les commercialiser. Il est très important de peaufiner le site afin de donner aux internautes l'envie d'acheter des vins qui puissent les faire rêver. Nous faisons référence ici aux photos des parcelles de vignes du Domaine du Puits de Compostelle. Les prestations offertes par les professionnels peuvent paraître coûteuses au premier abord, mais les retombées commerciales, induites par cette ouverture du marché, permettent un rapide amortissement. La presse papier joue encore un rôle important dans la reconnaissance des vignobles nivernais.

\section{B. La reconnaissance médiatique}

Les vins du Nivernais bénéficient d'une attention soutenue émanant de la presse. Les quotidiens régionaux - Le Journal du Centre, L'Yonne républicaine -, les hebdomadaires locaux - Le Charitois, Le Régional de Cosne - font régulièrement écho des nouvelles des vignobles nivernais. Les journalistes aiment faire des reportages sur un sujet convivial et apprécient l'accueil qui leur est réservé. Les vendanges occupent une place de choix dans les colonnes de la presse régionale. La presse spécialisée nationale n'est pas en reste, surtout si le vignoble dispose d'un leader charismatique, produisant de surcroît, des vins hors normes. Paulat, Hervé, par le mode d'expression original de leurs vins, sont applaudis par La Revue du Vin de France. Les hebdomadaires nationaux signent aussi des articles sur ces vignobles proches de Paris $(200 \mathrm{~km})$. Cependant, nous constatons la récurrence d'articles concernant le(s) vigneron(s) en vue et le manque de curiosité des journalistes face aux efforts réalisés par certains viticulteurs plus réservés, qui améliorent leurs modes de conduite en vigne et d'élevage en cave et obtiennent des résultats fort honorables. Si la médiatisation nationale de ces vignobles a un effet immédiat auprès des vignerons médiatisés (visites, commandes), elle a seulement un impact ponctuel. Il est donc nécessaire soit de réitérer les bonnes performances, soit de jouer la carte médiatique pour attirer régulièrement la presse à s'intéresser au domaine.

54 Certains guides sont très spécifiques, à l'image du Guide des Vins Biologiques. Une fois inventoriés par les journalistes, les domaines ont toutes les chances d'être cités les années suivantes. Les guides de référence œnologique, comme le Guide Hachette, ont une influence plus efficace. En effet, les connaisseurs, les touristes en villégiature dans la région, se réfèrent à ce guide apprécié pour son travail sérieux sur le terrain. Cependant, pour être retenu par le guide Hachette, le vigneron doit expédier des échantillons au centre régional de dégustation. Nous ne savons pas combien de viticulteurs nivernais ont fait la démarche. Toujours est-il que le Guide Hachette 2004 mentionne deux producteurs de Coteaux Charitois - le Domaine du Puits de Compostelle et le Domaine de la Vernière à Chasnay -et cinq de Coteaux du Giennois : Michel Langlois et le Domaine de Villargeau à Pougny, la SCEA Quintin Frères et le Lycée agricole à Cosne-sur-Loire, les Caves de Pouilly-surLoire. 
L'organisation de concours viticoles donne une chance supplémentaire aux domaines d'être connus et reconnus. Au premier Concours de Vins de Centre Loire, organisé à Bourges le 7 juin 2004, les Coteaux du Giennois ont reçu deux médailles d'or pour un Blanc 2003 du Lycée viticole de Cosne-sur-Loire, pour un Rouge 2003 des Caves de Pouilly. À l'occasion de cette manifestation, les vignobles de Sancerre et de Pouilly-sur-Loire ont raflé une grande partie des prix.

Les récompenses de rang international donnent une notoriété supplémentaire aux heureux élus. Ainsi, l'attribution de prix réitérés au Concours des Meilleurs Chardonnay du Monde a propulsé les Caves Tannaysiennes dans la sphère des vins appréciés par une clientèle qui aime le style des "bêtes à concours ", en l'occurrence, des vins prêts à boire ${ }^{13}$. La Cave des Coteaux Charitois a également été médaillée dans le cadre de ce même concours, qui compte parmi le jury plusieurs Anglo-Saxons, qui apprécient les Chardonnay boisés.

57 Les vignobles nivernais enregistrent une dynamique réelle depuis les années 1980-1990. Les progrès qualitatifs ont permis aux dossiers proposés à l'ONIVINS ou à l'INAO d'aboutir à la délimitation des espaces viticoles en aires de dénomination de Vins de Pays de zone ou en AOC. Cette démarche qualitative, remarquée et encouragée par les revues spécialisées et les guides, ouvre en grand les portes d'une commercialisation à une plus large échelle. Les viticulteurs, inscrits dans les aires de dénomination en Vins de Pays de zone,préparent l'étape suivante - obtenir la reconnaissance par l'INAO en VDQS -, bien que cela posera le problème de la concurrence avec acuité. Il leur faudra néanmoins être patients pour que leur dossier soit agréé par l'organisme national. Pour ces vignobles, une nouvelle page d'Histoire s'ouvrira alors.

\section{BIBLIOGRAPHIE}

Boulellal M. (2002). - « Grâce à eux, des vignobles ressuscitent ", La Revue du Vin de France, n 466 , p. 22.

Dupont J. (2002). — « Quand un vignoble au passé glorieux retrouve sa saveur ", Le Point, $\mathrm{n}^{\circ}$ 1536, p. 119.

Dupont J. (2005). - « Le vin de Benjamin ", Le Point, $\mathrm{n}^{\circ}$ 1693, p. 118.

Marlière A. (1990). - Clamecy et ses environs, Paris, Editions Res Universis, 492 p.

Guides spécialisés :

Guide Hachette 2004 (2003), Paris, Hachette Livre.

Guide des vins biologiques (2004), Paris, Editions Dervy.

Sans nom d'auteur (déc.2003-janv.2004), « L'instit à l'école de la vigne ", Bourgogne, n 53, p.77.

DVD :

Invitation à Tannay, DVD à paraître, fin 2005. 


\section{Sites internet consultés :}

http://www.puitsdecompostelle.com, 23 avril 2005.

http://www.1855.com/région, 23 avril 2005.

http://www.guy-saget.com, 24 avril 2005.

http://www.cavesdepouillysurloire.com, 24 avril 2005.

\section{NOTES}

1. Les œufs en meurette sont des œufs pochés dans une sauce au vin. Le tour de main nécessite de présenter le blanc cuit à point et le jaune encore moelleux.

2. Cette appellation a été choisie, car un puits se trouve sur le domaine. Au Moyen-âge, ce puits permettait aux pèlerins en route pour Saint-Jacques de se désaltérer. La région est en effet traversée par l'une des routes de pèlerinage passant par Vézelay, La Charité.

3. VDQS : Vin De Qualité Supérieure.

4. Cette appellation est liée à la présence de nombreux fossiles en forme de tête de chat. Il s'agit de calcaires coquilliers du Jurassique supérieur.

5. En 2004, la récolte est répartie de la manière suivante : $482 \mathrm{hl}$ de Chardonnay, $272 \mathrm{hl}$ de Melon, 114 hl de Pinot noir et 32 hl de Gamay.

6. Ils sont désormais 580 .

7. Elles se monnaient à 228 euros en 2005.

8. La Chablisienne est la cave coopérative de Chablis dans l'Yonne.

9. En 2005, ils sont compris entre 3,8 et 5,5 euros. La "Cuvée Jeunesse ", Les Belles Fornasses 2002, est vendue en fontaine à vin au prix de 38 euros les 10 litres. Les Têtes de Chat 1999 et Les Belles Fornasses 1998 sont commercialisées à 5,3 euros la bouteille, Les Belles Fornasses 1997 5,5 euros.

10. Il est ainsi possible de déguster les vins du Domaine de Villargeau dans plusieurs brasseries et cafés de Paris.

11. La déléguée commerciale, chargée de promouvoir les vins des Caves de Tannay, dans la Nièvre, l'Yonne et de Saône-et-Loire, a dû être licenciée en juin 2005.

12. Les tarifs sont échelonnés entre 4,5 euros pour un Sauvignon 2004 et 6,5 euros pour un Pinot noir 2004 élevé en fût de chêne.

13. Ce concours organisé par la Cave de Chaintré (Saône-et-Loire) a récompensé les Caves Tannaysiennes par trois fois : médaille de bronze en 2000 pour un 1998, médaille d'or en 2001 pour un 1999 et médaille d'argent en 2002 pour un 2000.

\section{RÉSUMÉS}

Depuis les années 1990, après une quasi extinction, les vignobles nivernais se reconstituent. L'appellation Pouilly-sur-Loire fait exception: elle s'est maintenue et développée à travers les décennies. Cette renaissance a été initiée par quelques personnes, viticulteurs de la jeune génération, néoruraux attirés par des contrées aptes à cultiver la vigne, des maires ou par des institutions bien implantées localement, comme le Crédit Agricole, qui ont insufflé une 
dynamique propre à développer des aires de production et des vins, reconnus par les organes viticoles officiels, les revues spécialisées, à l'instar de la Revue du Vin de France. Comment ces vignobles ont-ils pu émerger dans un contexte de plus en plus concurrentiel? Comment ont-ils $\mathrm{pu}$ attirer des consommateurs curieux de déguster et d'apprécier des vins aux caractères spécifiques? Quel rôle ont pu jouer les vignobles AOC de proximité: Sancerre et Pouilly sur Loire pour les vins AOC Coteaux du Giennois, les Vins de Pays des Coteaux Charitois et Vézelay pour les Vins de Pays des Coteaux de Tannay?

Since 1990, the vineyards of Nivernay have undergone a revival. (The appellation Pouilly-surLoire is an exception: she has been maintained and developed through the decades). This revival was initiated by many people: young wine growers, new country people, attracted by land suitable for viticulture, or well-established local institutions such as the rural bank, Crédit Agricole, who are energizing production areas and their wines. These vineyards and wines are recognized by official wine-growing organisations, and in specialized reviews like the Revue du Vin de France. How could these vineyards emerge in such a competitive environment? How could they attract the attention of enquiring consumers, to taste and appreciate wines of specific character? What role did AOC vineyards play in this revival: Sancerre and Pouilly-sur-Loire for AOC wines Coteaux of Giennois and Coteaux Charitois, Vézelay for the wines from the Coteaux de Tannay?

Seit den 90er Jahren sind die Weinberge im Nivernais wieder aufgelebt. Diese Wiedergeburt ist durch einige Personen oder Institutionen verursacht worden: junge Weinbauern, Neuankömmlinge, die in diesen Regionen leben, oder Banken - wie der «Crédit Agricole» -, die den neuen Weinbauern helfen können. Die Anbauflächen der Weinberge nehmen zu. Die offizielle Weinbauorganisationen, die spezialisierten Zeitschriften erkennen die Qualitäten dieser Weinberge an. Aber, wie können sich diese Weinberge in einer starken Konkurrenz behaupten? Wie können sie das Interesse der Verbraucher auf sich ziehen? Welche Rolle haben die näherliegenden Weinberge - Sancerre, Pouilly-sur-Loire -, für die westlichen Weinberge (Coteaux von Giennois, Coteaux Charitois), Vézealy für die Weinberge des Coteaux von Tannay?

\section{INDEX}

Mots-clés : dynamiques interne et externe, reconnaissance, renaissance, vignobles nivernais, Vins de Pays de zone

Schlüsselwörter : Auflebung, Erkennung, innerliche und äusserliche Dynamik, Länderweinberge, Weinberge im Nivernais

Keywords : internal and external ernergizing, landwineyards, Nivernais wineyards, recognition, revival

\section{AUTEUR}

\section{MARIE-PIERRE CERVEAU}

Docteur en géographie, Lycée R. Rolland, 58500 ClamecyINALCO, Bd Maréchal Delattre de Tassigny, 75016 Paris 